\title{
Fused CLEAN deconvolution for compact and diffuse emission
}

\author{
L. Zhang ${ }^{1,2}$ \\ 1 Guizhou University, Guiyang 550025, PR China \\ e-mail: lizhang. science@gmail.com \\ 2 CAS Key Laboratory of Solar Activity, National Astronomical Observatories, Beijing 100012, PR China
}

Received 24 March 2018 / Accepted 20 July 2018

\begin{abstract}
Context. CLEAN algorithms are excellent deconvolution solvers that remove the sidelobes of the dirty beam to clean the dirty image. From the point of view of the scale, there are two types: scale-insensitive CLEAN algorithms, and scale-sensitive CLEAN algorithms. Scale-insensitive CLEAN algorithms perform excellently well for compact emission and perform poorly for diffuse emission, while scale-sensitive CLEAN algorithms are good for both point-like emission and diffuse emission but are often computationally expensive. However, observed images often contain both compact and diffuse emission. An algorithm that can simultaneously process compact and diffuse emission well is therefore required.

Aims. We propose a new deconvolution algorithm by combining a scale-insensitive CLEAN algorithm and a scale-sensitive CLEAN algorithm. The new algorithm combines the advantages of scale-insensitive algorithms for compact emission and scale-sensitive algorithms for diffuse emission. At the same time, it avoids the poor performance of scale-insensitive algorithms for diffuse emission and the great computational load of scale-sensitive algorithms for compact emission in residuals.

Methods. We propose a fuse mechanism to combine two algorithms: the Asp-Clean2016 algorithm, which solves the computationally expensive problem of convolution operation in the fitting procedure, and the classical Högbom CLEAN (Hg-Clean) algorithm, which is faster and works equally well for compact emission. It is called fused CLEAN (fused-Clean) in this paper.

Results. We apply the fused-Clean algorithm to simulated EVLA data and compare it to widely used algorithms: the $\mathrm{Hg}$-Clean algorithm, the multi-scale CLEAN (Ms-Clean), and the Asp-Clean2016 algorithm. The results show that it performs better and is computationally effective.
\end{abstract}

Key words. methods: data analysis - techniques: image processing

\section{Introduction}

For many reasons, such as technical difficulties, the size of a single dish is severely limited, as is its resolution. Interferometric measurements with some imaging techniques such as deconvolution can go beyond the resolution limitation of a single telescope. Radio telescope arrays currently often use tens of telescopes to measure visibilities. However, the sampling is incomplete. These missing spatial frequencies lead to a dirty image, which is the corrupted version of the true sky image. Deconvolution algorithms are used to remove the effects of the corruption to obtain the restored image.

The most widely used deconvolution algorithms in the radio synthesis imaging field are CLEAN algorithms. Scaleinsensitive CLEAN algorithms decompose the true sky image as a collection of point sources or scaled delta functions. The original algorithm (Högbom 1974) has been proposed by Högbom in the 1970s. It is a matched-pursuit algorithm and employs iterations to approximate the true sky image. For point-like emission, it is quite good and speedy enough. However, it needs a huge number of scaled delta functions to approximate diffuse emission and complex images, and it lacks a mechanism to introduce the dependence among the neighboring points. Thus the residuals of extended sources often include some significantly correlated structures.

Scale-sensitive CLEAN algorithms (Bhatnagar \& Cornwell 2004; Cornwell 2008) introduce a priori the knowledge that the true image is composed of different spatial scales. The pixels within one scale are dependent, which can constrain the unsampled spatial frequencies. Extended emission can be represented with a small number of components in scale-sensitive CLEAN algorithms. Even though some papers (Zhang et al. 2016a,b) have improved scale-sensitive CLEAN algorithms, they are still not computationally efficient for compact emission.

So far, these deconvolution algorithms are designed for either compact or diffuse emission. However, observations contain both compact and diffuse emission within the field of view, which requires an algorithm that can process both compact and diffuse emission well. To solve the problem, we propose an efficient algorithm that combines Asp-Clean2016 and Hg-Clean. This is not a direct combination of the two algorithms, but a more sophisticated way; it only combines in the phase of the component search in the minor cycle and uses the same major cycle.

The paper is structured as follows. In Sect. 2 we describe the imaging theory, the $\mathrm{Hg}$-Clean algorithm, and the Asp-Clean2016 algorithm. In Sect. 3 we describe the fused-Clean algorithm in detail. In Sect. 4 we give some examples and compare our results to other classical algorithms. In Sect. 5 we discuss this algorithm and conclude.

\section{Imaging theory and CLEAN algorithms}

To understand our algorithm, here we introduce the radio interferometric imaging theory and two algorithms that are used in the proposed algorithm. 


\subsection{Imaging theory}

In the van Cittert-Zernike theorem (Thompson et al. 2017), the true visibility function and the sky brightness function are a pair of Fourier transformations. The true visibilities are $V^{\text {true }}$

$V^{\text {true }}=F I^{\text {true }}$,

where $F$ is the Fourier transformation and $I^{\text {true }}$ is the sky brightness function. This is an ideal and continuous case. The true image can be recovered by directly employing inverse Fourier transformation. For real cases, however, the sample is incomplete and noisy. The measured visibilities $V^{\text {measured }}$

$V^{\text {measured }}=S F I^{\text {true }}+S N$,

where $S$ is the sampling function and $N$ is the noise in Fourier domain. Here we ignore these operations of weighting, convolutional interpolation, and resampling, and express the dirty image $I^{\text {dirty }}$ as

$I^{\text {dirty }}=F^{-1} S F I^{\text {true }}+F^{-1} S N$,

where $F^{-1}$ is the inverse Fourier transform operation. In the convolution theorem of Fourier transform theory, the dirty image $I^{\text {dirty }}$ can be expressed as

$I^{\text {dirty }}=B^{\text {dirty }} I^{\text {true }}+B^{\text {dirty }} N$,

where $B^{\text {dirty }}$ is a Toeplitz matrix that is composed of the shifted dirty beams. Because of the incomplete sampling, the dirty beam often has many non-ignorable wide-spread sidelobes. Deconvolution is a solver that removes the effect of these dirty beam sidelobes.

\section{2. $\mathrm{Hg}$-Clean algorithm}

The Hg-Clean algorithm decomposes the true sky brightness as a set of scaled delta functions,

$I^{\text {model }}=\sum_{\mathrm{n}} I_{\mathrm{n}}^{\mathrm{amp}} \delta_{\mathrm{n}}\left(x-x_{\mathrm{n}}, y-y_{\mathrm{n}}\right)$,

where $I_{\mathrm{n}}^{\mathrm{amp}}$ is the amplitude of the $n$th component and $\delta_{\mathrm{n}}$ is the delta function in the position $\left(x_{\mathrm{n}}, y_{\mathrm{n}}\right)$. The error of the component estimation in the minor cycle is corrected in the fused-Clean algorithm by updating residual visibilities from the original visibility data in the major cycle. Very many components are needed to represent a large diffuse emission. However, the computational load of the algorithm for each component is small. This is effective for compact emission.

\subsection{Asp-Clean2016 algorithm}

The Asp-Clean2016 algorithm (Zhang et al. 2016a) is an efficient implementation of the Asp-Clean algorithm (Bhatnagar \& Cornwell 2004). It parameterizes the true sky image as a collection of circular Gaussian functions with different scales,

$I^{\text {model }}=\sum_{\mathrm{n}} G_{\mathrm{n}}\left(\alpha_{\mathrm{n}}, x_{\mathrm{n}}, y_{\mathrm{n}}, \sigma_{\mathrm{n}}\right)$,

where

$G\left(\alpha_{\mathrm{n}}, x_{\mathrm{n}}, y_{\mathrm{n}}, \sigma_{\mathrm{n}}\right)=\alpha_{\mathrm{n}} \exp \left(-\frac{1}{2} \frac{\left(x-x_{\mathrm{n}}\right)^{2}+\left(y-y_{\mathrm{n}}\right)^{2}}{\sigma_{\mathrm{n}}^{2}}\right)$, where $\alpha_{\mathrm{n}}$ is the amplitude of the $n$th component, $\sigma_{\mathrm{n}}$ is the scale of the Gaussian component, and $x_{\mathrm{n}}$ and $y_{\mathrm{n}}$ are the parameters of positions.

The Asp-Clean algorithm finds the best-fit scale components with active sets by minimizing the objective function $\chi^{2}$ for each component

$\chi^{2}=\left\|I_{\mathrm{n}-1}^{\text {residual }}-B^{\text {dirty }} I_{\mathrm{n}}^{\text {model }}\right\|_{2}^{2}$,

where $I_{\mathrm{n}}^{\text {residual }}$ is the residual image in $(n-1)$ th iteration, $I_{\mathrm{n}}^{\text {model }}$ is the current model component, and $\|\cdot\|_{2}$ is the Euclidean norm. Since the convolution is in the component-fitting objective function, the Asp-Clean algorithm is computationally expensive. It is removed to speed up the process by analytically computing model components in the Asp-Clean2016 algorithm (Zhang et al. 2016a). The procedure of the Asp-Clean2016 algorithm is similar to the Asp-Clean algorithm, but it minimizes a different objective function of the fitting part,

$\chi^{2}=\left\|I_{\mathrm{n}}^{\text {residual }}-I_{\mathrm{n}}^{\text {gauss }}\right\|_{2}^{2}$,

where $I_{\mathrm{n}}^{\text {gauss }}$ is a Gaussian component that was fitted from the residual image. This is very efficient for diffuse emission (Zhang et al. 2016a). However, it is still time-consuming for compact emission.

\section{Fused-Clean algorithm}

To achieve the goal of simultaneously processing both compact and diffuse emission well, the fused-Clean algorithm combines the advantages of the different algorithms and can automatically trigger different algorithms in different situations to speed up and improve the performance of the algorithm. The basic procedure is as follows.

1. Smooth residual image $I_{\mathrm{n}}^{\text {residual }}$ (the dirty image for the first time) with $s$ scales.

2. Find peaks from these smoothed residual images $I_{\mathrm{n}, \mathrm{s}}^{\text {residual }}$; the global peak $G_{1}\left(a_{1}, x_{1}, y_{1}, \sigma_{1}\right)$ is used as the initial guess of the current parameters.

3. Trigger an algorithm according to the current situation.

4. Find new parameters of the current model component $I_{\mathrm{n}}^{\text {component }}$.

5. Update the model image $I_{\mathrm{n}}^{\text {model }}=I_{\mathrm{n}-1}^{\text {model }}+I_{\mathrm{n}}^{\text {component }}$.

6. Calculate the residual image $I_{\mathrm{n}}^{\text {residual }}=I_{\mathrm{n}-1}^{\text {residual }}-B^{\text {dirty }} I_{\mathrm{n}}^{\text {model }}$.

7. Iterate until one of the termination criteria is satisfied.

8. Compute the restored image $I^{\text {restored }}=B^{\text {clean }} I_{\mathrm{m}}^{\text {model }}+I_{\mathrm{m}}^{\text {residual }}$ with the restored beam $B^{\text {clean }}$ after $m$ iterations.

All model components are found by equivalently minimizing the objective function in the major cycle,

$\chi^{2}=\left\|V^{\text {measured }}-S F^{-1} I^{\text {model }}\right\|_{2}^{2}$,

It can find the best fit to the measured visibilities.

In the beginning phase of deconvolution, the matchedfiltering technique is used to find the initial position and scale of the strongest emission in the same way as in the Asp-Clean2016 algorithm. If the initial scale is larger than the scale of the dirty beam, then the minor cycle of the Asp-Clean2016 algorithm is triggered once. The optimal scale and position are found by explicitly minimizing the objective function,

$\chi^{2}=\left\|I_{\mathrm{n}}^{\text {residual }}-I_{\mathrm{nb}}^{\text {gauss }}\left(\alpha_{\mathrm{nb}}, x_{\mathrm{nb}}, y_{\mathrm{nb}}, \omega_{\mathrm{nb}}\right)\right\|_{2}^{2}$, 
where $I_{\mathrm{nb}}^{\text {gauss }}$ is a Gaussian function with parameters (amplitude $\alpha_{\mathrm{nb}}$, location $x_{\mathrm{nb}}, y_{\mathrm{nb}}$, and width $\left.\omega_{\mathrm{nb}}\right)$. The updated direction is estimated by computing the gradient of $\chi^{2}$ with respect to the parameters $p_{\mathrm{nb}}$ given by

$$
\frac{\partial \chi^{2}}{\partial p_{\mathrm{nb}}}=\frac{\partial \chi^{2}}{\partial I_{\mathrm{nb}}^{\text {gauss }}} \frac{\partial I_{\mathrm{nb}}^{\text {gauss }}}{\partial p_{\mathrm{nb}}}=-2\left[I_{\mathrm{n}}^{\text {residual }}\right]^{\mathrm{T}} \frac{\partial I_{\mathrm{nb}}^{\text {gauss }}}{\partial p_{\mathrm{nb}}}
$$

where $p_{\mathrm{nb}} \equiv\left\{\alpha_{\mathrm{nb}}, x_{\mathrm{nb}}, y_{\mathrm{nb}}, \omega_{\mathrm{nb}}\right\}$. For the second-order optimization method (e.g., the Levenberg-Marquardt algorithm Marquardt 1963), we also need to compute or approximate the Hessian matrix. After converging to the solution of $I_{\mathrm{nb}}^{\text {gauss }}$, the parameterized component can be analytically computed as

$\omega_{\mathrm{n}}=\sqrt{\omega_{\mathrm{nb}}^{2}-\omega_{\mathrm{b}}^{2}}$,

where $\omega_{\mathrm{n}}, \omega_{\mathrm{nb}}, \omega_{\mathrm{b}}$ are the widths of the current model component $I_{\mathrm{n}}^{\text {component }}, I_{\mathrm{nb}}^{\text {gauss }}$ and the Gaussian beam approximated from the dirty beam, respectively. The amplitude $\alpha_{\mathrm{n}}$ of $I_{\mathrm{n}}^{\text {component }}$ is computed as

$\alpha_{\mathrm{n}}=\frac{\alpha_{\mathrm{nb}} \omega_{\mathrm{nb}}^{2}}{2 \pi \alpha_{\mathrm{b}} \omega_{\mathrm{b}}^{2} \omega_{\mathrm{n}}^{2}}$,

where $\alpha_{\mathrm{b}}$ and $\alpha_{\mathrm{nb}}$ are the amplitudes of the Gaussian beam and $I_{\mathrm{nb}}^{\text {gauss }}$, respectively.

Explicitly, minimization optimizes each component, which is a best fit for the current residuals. The Asp-Clean2016 algorithm (Zhang et al. 2016a) has shown that the analytical computation of components is very efficient for diffuse emission.

When the initial scale estimated by matched-filtering technique is smaller than a threshold (e.g., 1.2 times the width of the dirty beam) or very small scale components frequently appear in the last iterations (e.g., the FWHM of five components are smaller than 1 pixel in the last ten components), then the minor cycle of the $\mathrm{Hg}-\mathrm{Cl}$ ean algorithm will be triggered. Compared to scale-sensitive CLEAN algorithms, the Hg-Clean is more efficient for compact emission. It does not perform an explicit fitting, but determines the peak of the current residuals and then subtracts a scaled version of the dirty beam from the current residuals. In other words, the updated direction is estimated by finding the peak of the current residuals,

$\frac{\partial \chi^{2}}{\partial p_{\mathrm{n}}} \equiv \frac{\partial \chi^{2}}{\partial I_{\mathrm{n}}^{\text {amp }}}=-2\left[I_{\mathrm{n}}^{\text {residual }}\right]^{\mathrm{T}} \frac{\partial p_{\mathrm{n}}}{\partial I_{\mathrm{n}}^{\text {amp }}}=-2 I_{\mathrm{n}, \mathrm{x}_{\mathrm{n}}, y_{\mathrm{n}}}^{\text {residual }}$,

where $p_{\mathrm{n}}=I_{\mathrm{n}}^{\mathrm{amp}}$ and $I_{\mathrm{n}, \mathrm{x}_{\mathrm{n}}, y_{\mathrm{n}}}^{\text {residual }}$ is the peak point located at $\left(x_{\mathrm{n}}, y_{\mathrm{n}}\right)$ in the $n$th iterations. The iterative search for $I_{\mathrm{n}}^{\text {amp }}$ and then the shift-and-subtract operation in the $\mathrm{Hg}$-Clean algorithm is equivalent to a fast implementation of the minimization of the objective function given by Eq. (8). When the Hg-Clean is triggered, it will be ran for several times. In practice, more compact emission will appear in the residuals when the deconvolution reaches deeper, so that a monotonic function for the triggering number can process it well. We have found the following relation to work well:

$t_{\text {tn }}=100+50 *\left(\mathrm{e}^{0.05 * t n}-1\right)$.

$t_{\mathrm{tn}}$ is the times of executing $\mathrm{Hg}$-Clean when $\mathrm{Hg}$-Clean is triggered $t n$th times. The specific form of this function in Eq. (16) is not important, but with the increase of the triggering times for the $\mathrm{Hg}$-Clean algorithm, the function should be increasing.
In the fused-Clean algorithm, the $\mathrm{Hg}$-Clean algorithm is used for compact emission and the Asp-Clean2016 algorithm is used for diffuse emission. The scale adaptivity of the Asp-Clean2016 algorithm can separate emission and noise, while the Hg-Clean algorithm is efficient for compact emission. In the fused-Clean algorithm, the two algorithms are triggered alternately, so that emission and noise are effectively separated.

\section{Numerical experiment and comparison}

In this section, we apply the fused-Clean algorithm to the EVLA $^{1}$ simulated data to evaluate its performance and compare it to these frequently used CLEAN algorithms: the Hg-Clean algorithm, the Ms-Clean algorithm, and the Asp-Clean2016 algorithm. The test M31 image is shown in Fig. 1a, and its brightness ranges from $0 \mathrm{Jy}_{\mathrm{pixel}}{ }^{-1}$ to $0.1 \mathrm{Jy} \mathrm{pixel}^{-1}$. We performed a simulated observation with the $\mathrm{B}$ configuration of EVLA using the CASA software ${ }^{2}$. The observation was made in $\mathrm{L}$ band with a bandwidth of $1 \mathrm{GHz}$ and 32 channels, and it lasted six hours. Gaussian white noise was added to the "measured" visibilities, which means that the dirty image has a noise level of RMS $5 \times 10^{-5} \mathrm{Jy}$. The resolution of the images was $1^{\prime \prime}$ and the width of the main lobe of the dirty beam was about $2^{\prime \prime}$. The corresponding dirty image with the robust $(=0)$ weighting is shown in Fig. 1b, where the data range reaches from $-0.039 \mathrm{Jy} \mathrm{pixel}^{-1}$ to $0.61 \mathrm{Jy} \mathrm{pixel}^{-1}$.

The deconvolution results are shown in Fig. 1. The model image displayed in Fig. 1c is composed of 155 extended components and 20196 compact components, which can represent the true emission well. No significant signal in the residual image displayed in Fig. 1d indicates that the fused-Clean deconvolution can extract the signal fully, and signal and noise are effectively separated.

These scale choices of deconvolving the M31 image and the change of the model flux with iterations are displayed in Fig. 2, which contains only the first 100 iterations and the last 10000 iterations for effective visualization. The choices of algorithms can be known through the choices of the component scale sizes. If the scale size is greater than zero, then the Asp-Clean2016 algorithm is selected; otherwise the Hg-Clean algorithm is selected. We can know the deconvolution behaviors of the fused-Clean algorithm from Fig. 2. 1) Most flux was recovered in the beginning phase of the deconvolution. The main emission is reconstructed in the first $\sim 50$ iterations. AspClean2016 is more frequently executed in the beginning phase because Asp-Clean2016 has more sparse representation capacity for diffuse emission. 2) Most iterations were used to reconstruct weak and small-scale emission. This compact emission corresponds to compact sources or broken emission from an inaccurate representation of diffuse emission. $\mathrm{Hg}$-Clean is required frequently to deconvolve this compact emission to approximate the latent true image. 3) After triggering the Hg-Clean algorithm, some weak and large-scale emission may appear and can be reconstructed effectively by the Asp-Clean2016 algorithm. 4) We did not set the iteration number for the Asp-Clean2016 and Hg-Clean algorithms. The 155 Asp-Clean2016 and $20196 \mathrm{Hg}-$ Clean deconvolutions are completely determined by the scale complexity of the dirty image and by the parameters of the deconvolution algorithm. This also shows the adaptive capacity of calling these two sub-algorithms.

\footnotetext{
1 http://www.aoc.nrao.edu/evla/

2 http://casa.nrao.edu/
} 

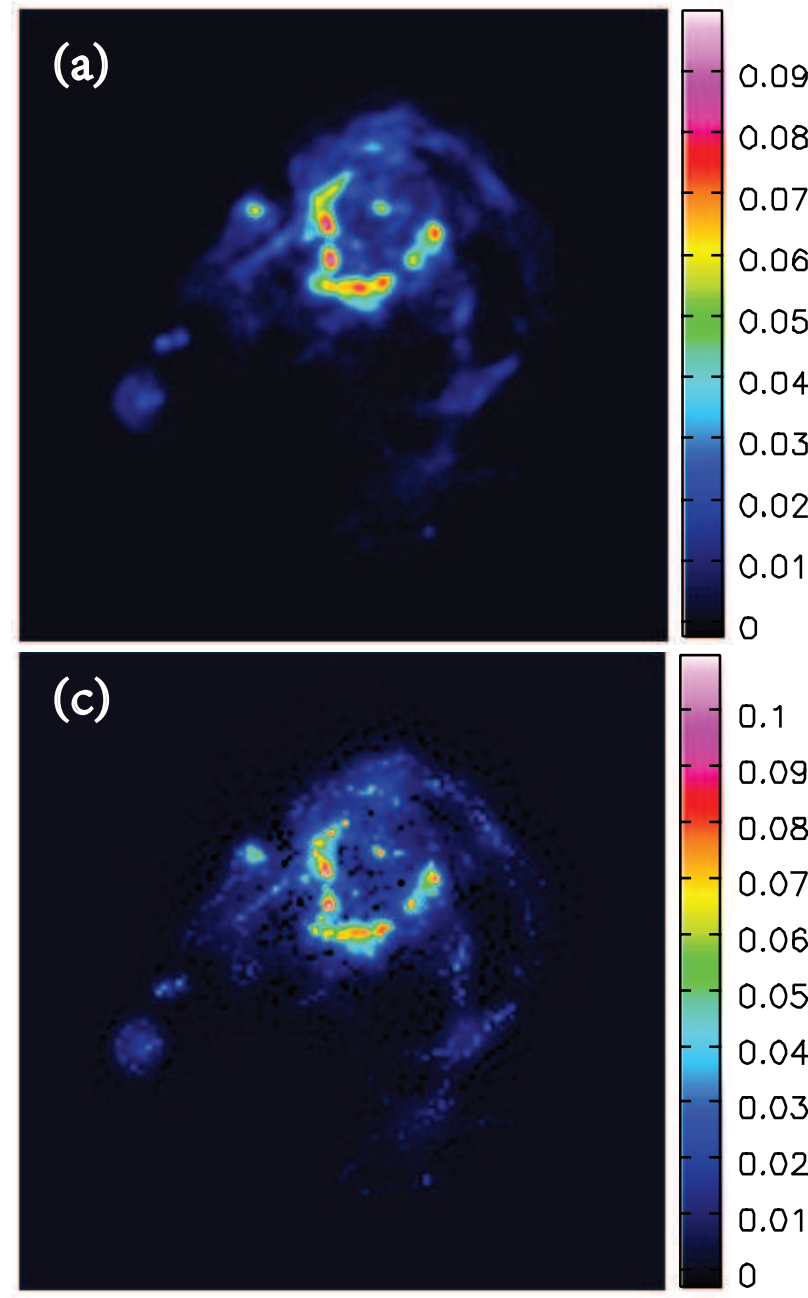
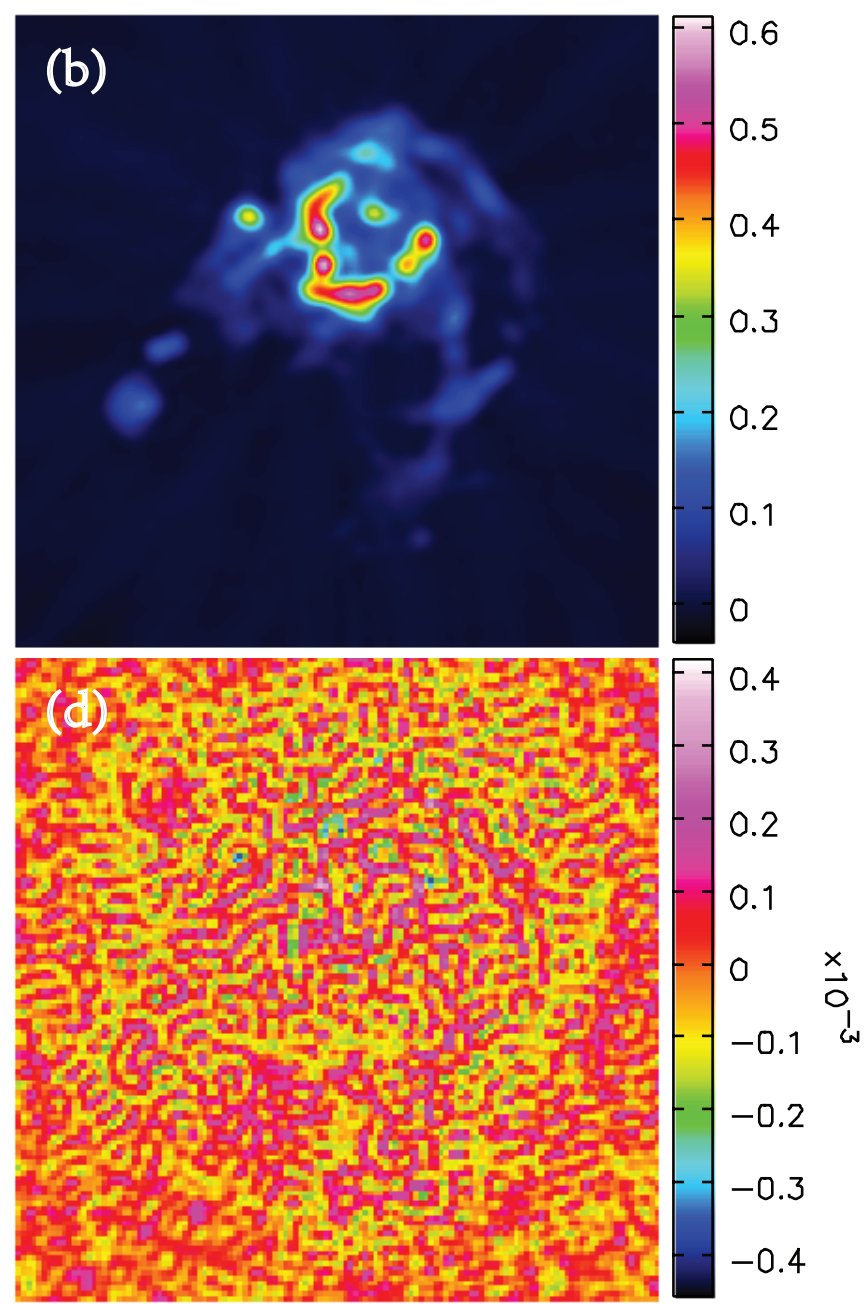

Fig. 1. Results of simulated EVLA observations of the M31 image deconvolved by the fused-Clean algorithm. Panel a: original image; panel $b$ : dirty image with Briggs weighting; panel $c$ : model image; and panel $d$ : residual image.
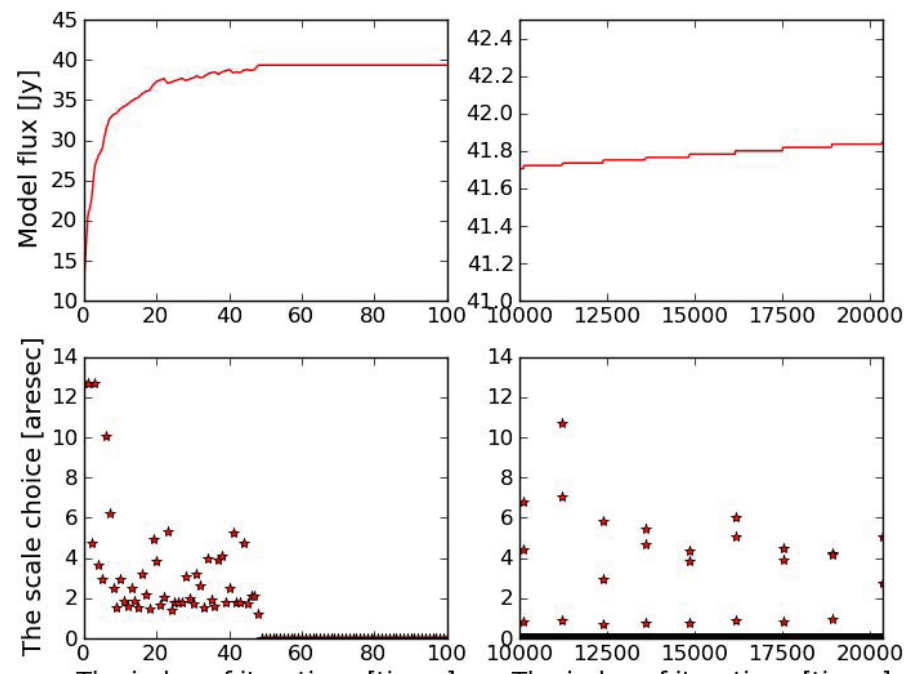

The index of iterations [times]

Fig. 2. Scale choices and model flux for deconvolving the dirty M31 image with fused-Clean.

In short, diffuse emission is recovered with the AspClean2016 algorithm, and compact emission is reconstructed with the Hg-Clean algorithm. The Asp-Clean2016 algorithm is almost always used preferentially when the residual image

contains much signal. As the deconvolution continues, the signals in the residual image will decrease and much scale-less emission will appear. Then the $\mathrm{Hg}$-Clean algorithm dominates the deconvolution process.

To compare the performance of the fused-Clean algorithm with other typical CLEAN deconvolution algorithms, the corresponding deconvolution results are displayed in Fig. 3 and listed in Table 1. The model images displayed in Fig. 3a0 from the $\mathrm{Hg}$-Clean algorithm are composed of 100000 compact components, and the corresponding residual image in Fig. 3a1 contains many correlated features because delta function cannot physically represent diffuse emission well. The model image from the Ms-Clean algorithm that uses enumeration scales has 2000 components and less signal in the residuals. The AspClean2016 and fused-Clean algorithms use adaptive scales. They can represent an image more sparsely than the previous two algorithms. The fused-Clean algorithm combines the Asp-Clean2016 algorithm with the Hg-Clean algorithm, which represents compact emission more effectively. This can separate signal and noise more effectively. No significant signal is in the residual image displayed in Fig. 3d1 from the fused-Clean algorithm. The fused-Clean deconvolution has the highest dynamic range (defined in Li et al. 2011) in this experiment from Table 1. All these results show that the performance of the fused-Clean algorithm is excellent, which can be also proved by the numerical comparison in Table 1. 

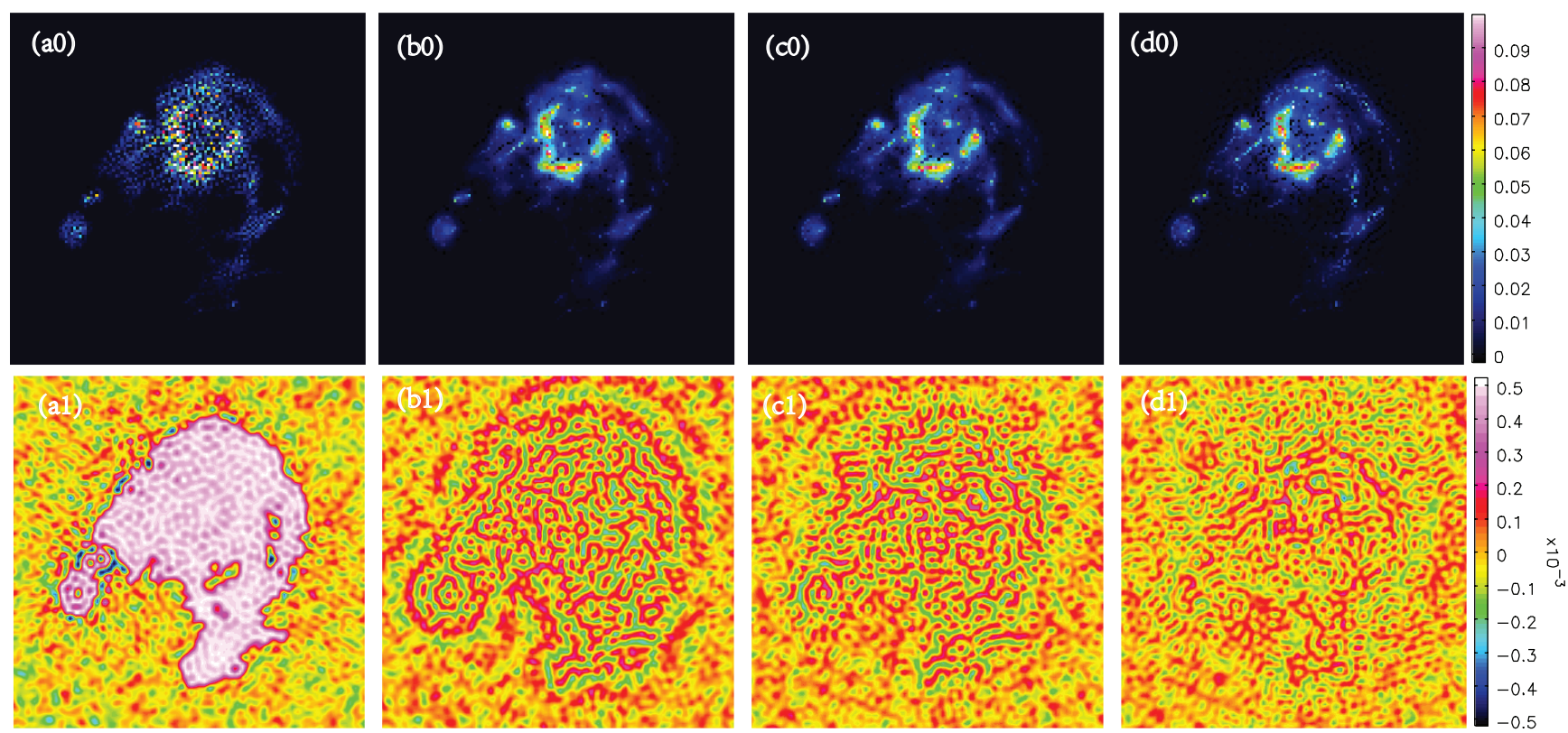

Fig. 3. Deconvolution results of the M 31 image. From left to right, columns: Hg-Clean, Ms-Clean, Asp-Clean2016, and fused-Clean. From top to bottom, rows: model images and the residual images.

Table 1. Numerical comparison of different deconvolution algorithms for the "M 31 " simulation.

\begin{tabular}{ccccc}
\hline & $\mathrm{Hg}$ & $\mathrm{Ms}$ & Asp2016 & Fused \\
\hline NCC & 100000 & - & - & 20196 \\
NDC & 0 & 20000 & 3500 & 155 \\
DR & 2763 & 5706 & 6083 & 6811 \\
\hline
\end{tabular}

Notes. NCC, NDC, and DR are short for the number of compact components, the number of diffuse components in these model images, and the dynamic ranges, respectively. Some compact components may be in these model images deconvolved with the Ms-Clean and AspClean2016 (here Asp2016) algorithms, but it is hard to count them. They are shown by the dash.

It is worth mentioning that the fused-Clean algorithm is more robust and faster than the Asp-Clean2016 algorithm. A combination of the $\mathrm{Hg}$-Clean algorithm equivalently introduces a new scheme to jump out of the possible local optimum in the AspClean2016 algorithm. In the M31 simulation, the fused-Clean deconvolution took about $4 \mathrm{~min}$, which is about four times faster than the Asp-Clean2016 algorithm. Experiments were ran on a typical graphics workstation. The speed increase arises because the Hg-Clean algorithm was used to represent compact emission. In the Asp-Clean2016 algorithm, finding a compact component from the current residual image needs to be explicitly fitted. This is achieved through iterative optimization, which is time consuming. However, finding a compact component through the $\mathrm{Hg}$-Clean algorithm only requires identifying the maximum value in the current residual image and some simple operations.

\section{Discussion and summary}

The fused-Clean introduces a good algorithm framework and the thought of algorithm union. In other words, this is a general method that can be applied to more algorithmic combinations than a mere combination of the Hg-Clean aglorithm and the Asp-Clean2016 algorithm. Combined algorithms can combine the advantages of different algorithms without maintaining their disadvantages. It performs excellently well, which is difficult for a single algorithm. In addition, if a combined algorithm can reduce the total computation complexity, then it is very helpful that a deconvolution algorithm can be developed into software. For this purpose, many scaleinsensitive and scale-sensitive algorithms can be combined to speed up the deconvolution process. For example, the Ms-Clean (Cornwell 2008) or MTMFS (Rau \& Cornwell 2011) can be combined with a scale-insensitive algorithm such as the Clark CLEAN (Clark 1980).

An algorithm combination should consider intrinsic factors and relations of model decomposition among iterations properly. In other words, a combined algorithm should be not a simple mechanical concatenation. A simple mechanical concatenation may not be sufficient to improve the performance.

Compressive-sensing based deconvolution algorithms make the obvious assumption that the signal is sparse in a certain domain. The CLEAN-based algorithm does not make such an assumption. Therefore, the performance of CLEAN-based algorithms (e.g., runtime and fidelity) is more stable when conditions change (see Li et al. 2011). At the same time, the fusedClean algorithm is naturally applicable to the CLEAN algorithm framework, that is, to minor cycle and major cycle (it is also implemented in the standard minor and major cycles) and also integrates well with some other typical synthesis imaging techniques, such as wide-field corrections.

The minor cycle of the fused-Clean contains the component estimation methods of the Asp-Clean2016 and the $\mathrm{Hg}$ Clean algorithms. The Asp-Clean2016 algorithm employs an analytical way to significantly reduce the computational load and at the same time keeps the excellent performance of adaptive scale deconvolution. The advantage of the $\mathrm{Hg}$-Clean algorithm is that it is excellent and fast for compact emission, but its disadvantage is that it is slow and difficult to fully represent for diffuse emission. The fused-Clean algorithm 
combines the speed and excellent performance of the AspClean2016 algorithm for diffuse emission with the speed of the $\mathrm{Hg}$-Clean algorithm for compact emission, and it avoids the slow speed of the Asp-Clean2016 algorithm for compact emission and the poor performance for diffuse emission of the $\mathrm{Hg}-\mathrm{Clean}$ algorithm.

Tests show that the performance of the fused-Clean algorithm is better than these typical CLEAN-based deconvolution algorithms. The algorithm is implemented with the CASA and Python language. The work to build it as an available deconvolution algorithm into the CASA software package is currently ongoing.

Acknowledgements. We thank the people who worked and are working on the Python and CASA projects, which provide an excellent development and simulation environment. The work is supported by the Open Research Program of the
CAS Key Laboratory of Solar Activity (KLSA201805) and the Guizhou Science \& Technology Cooperation Project-Talent Platform ([2017]5788).

\section{References}

Bhatnagar, S., \& Cornwell, T. J. 2004, A\&A, 426, 747

Bhatnagar, S., Rau, U., Green, D. A., \& Rupen, M. P. 2011, ApJ, 739, L20

Clark, B. G. 1980, A\&A, 89, 377

Cornwell, T. J. 2008, IEEE J. Sel. Top. Signal Process, 2, 793

Högbom, J. A. 1974, A\&AS, 15, 417

Li, F., Cornwell, T. J., \& de Hoog, F. 2011, A\&A, 528, A31

Marquardt, D. W. 1963, J. Soc. Ind. Appl. Math., 11, 431

Rau, U., \& Cornwell, T. J. 2011, A\&A, 532, A71

Schwab, F. R., \& Cotton, W. D. 1983, AJ, 88, 688

Thompson, A. R., Moran, J. M., \& Swenaon, G. W. 2017, Interferometry and Synthesis in Radio Astronomy, 3rd edn. (Switzerland: Springer International Publishing)

Zhang, L., Bhatnagar, S., Rau, U., \& Zhang, M. 2016a, A\&A, 592, A128

Zhang, L., Zhang, M., \& Liu, X. 2016b, Ap\&SS, 361, 153 